\title{
Gender issues in fundamental physics: Strumia's bibliometric analysis fails to account for key confounders and confuses correlation with causation
}

an open access journal

\section{Check for updates}

Citation: Ball, P., Britton, T. B., Hengel, E., Moriarty, P., Oliver, R. A., Rippon, G., Saini, A., \& Wade, J. (2021). Gender issues in fundamental physics: Strumia's bibliometric analysis fails to account for key confounders and confuses correlation with causation. Quantitative Science Studies, 2(1), 263-272. https://doi.org/10.1162 /qss_a_00117

DOI:

https://doi.org/10.1162/qss_a_00117

Corresponding Authors:

Erin Hengel

erin.hengel@liverpool.ac.uk

Philip Moriarty

philip.moriarty@nottingham.ac.uk
Copyright: () 2021 Philip Ball, T. Benjamin Britton, Erin Hengel, Philip Moriarty, Rachel A. Oliver, Gina Rippon, Angela Saini, and Jessica Wade. Published under a Creative Commons Attribution 4.0 International (CC BY 4.0) license.

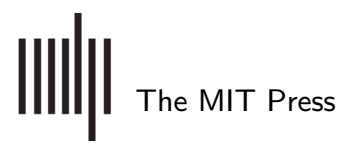

\author{
Philip Ball ${ }^{1}$, T. Benjamin Britton ${ }^{2}(\mathbb{D})$ Erin Hengel ${ }^{3}(\mathbb{D})$, Philip Moriarty $^{4}(\mathbb{D})$ Rachel A. Oliver $^{5}(\mathbb{D}$, \\ Gina Rippon ${ }^{6}$ (i) , Angela Saini ${ }^{7}$, and Jessica Wade ${ }^{8}$ (i) \\ ${ }^{1}$ Freelance Writer, London \\ ${ }^{2}$ Department of Materials, Imperial College London, London, SW7 2AZ \\ ${ }^{3}$ Department of Economics, University of Liverpool Management School, Liverpool, L69 7ZH \\ ${ }^{4}$ School of Physics \& Astronomy, University of Nottingham, Nottingham NG7 2RD, UK \\ ${ }^{5}$ Department of Materials Science, University of Cambridge, 27 Charles Babbage Road, Cambridge CB3 OFS \\ ${ }^{6}$ Professor Emeritus of Cognitive Neurolmaging, Brain Centre, Aston University, Birmingham B4 7ET \\ ${ }^{7}$ Science Journalist, London \\ ${ }^{8}$ Department of Physics, Imperial College London South Kensington Campus, London SW7 2AZ
}

\section{INTRODUCTION}

Alessandro Strumia recently published a survey of gender differences in publications and citations in high-energy physics (HEP). In addition to providing full access to the data, code, and methodology, Strumia (2021) systematically describes and accounts for gender differences in HEP citation networks. His analysis points both to ongoing difficulties in attracting women to HEP and an encouraging — though slow — trend in improvement.

Unfortunately, however, the time and effort that Strumia (2021) devoted to collating and quantifying the data are not matched by a similar rigor in interpreting the results. To support his conclusions, he selectively cites available literature and fails to adequately adjust for a range of confounding factors. For example, his analyses do not consider how unobserved factors-for example, a tendency to overcite well-known authors-drive a wedge between quality and citations and correlate with author gender. He also fails to take into account many structural and nonstructural factors-including, but not limited to, direct discrimination and the expectations that women form (and actions they take) in response to it - that undoubtedly lead to gender differences in productivity.

We therefore believe that a number of Strumia's conclusions are not supported by his analysis. Indeed, we reanalyze a subsample of solo-authored papers from his data, adjusting for year and journal of publication, authors' research age and their lifetime "fame." Our reanalysis suggests that female-authored papers are actually cited more than male-authored papers. This finding is inconsistent with the "greater male variability" hypothesis that Strumia (2021) proposes to explain many of his results.

In the conclusion to his paper, Strumia states that "... dealing with complex systems, any simple interpretation can easily be incomplete ...". We agree entirely. Strumia's simple-and, more importantly, simplistic_-analysis and interpretation are far from complete. 


\section{BIASED LITERATURE REVIEW}

Strumia (2021) notes that there is a "vast literature" dealing with gender differences in STEM subjects. Scientific analyses of gender differences should represent this literature in an evenhanded and unbiased manner; as Del Giudice, Puts et al. (2019) highlight, "An honest, sophisticated public debate on sex differences demands a broad perspective with an appreciation for nuance and full engagement with all sides of the question." That appreciation for nuance and full engagement is not present in Strumia (2021). For example

- In the introduction to his paper, Strumia asserts that "No significant biases have been found in examined real grant evaluations [Ceci et al., 2014; Ley \& Hamilton, 2008; Marsh, Jayasinghe, \& Bond, 2011; Mutz, Bornmann, \& Daniel, 2012] and referee reports of journals [Borsuk, Aarssen et al., 2009; Ceci et al., 2014; Edwards, Schroeder, \& Dugdale, 2018]." Yet a large body of literature-which he fails to cite-reaches the opposite conclusion. (See, for example, Burns, Straus et al., 2019; Card, DellaVigna et al., 2020; Dworkin, Linn et al., 2020; Fox \& Paine, 2019; Hengel, 2017; Helmer, Schottdorf et al., 2017; Royal Society of Chemistry, 2019; Steinberg, Skae, \& Sampson, 2018; Witteman, Hendricks et al., 2019 and references therein.) Strumia (2021)'s lack of balance in citing relevant work is misleading and arguably disingenuous. An objective analysis of gender differences should aim to be neither.

- Strumia (2021) also notes that theoretical modeling of citations is "affected by questionable systematic issues." We assume the use of "questionable" here is meant to capture relationships that are difficult to identify and quantify (e.g., due to a lack of suitable controls). We agree entirely that all efforts to study citations must be mindful of these limitations. Again, however, Strumia selectively cites just one paper that "[tries] to correct for some social factors," namely Caplar, Tacchella, and Birrer (2017). Other studies analyzing more restricted samples have come to the opposite conclusion (e.g., Card et al., 2020; Hengel \& Moon, 2020).

We do not, of course, expect Strumia (2021) to review the entire research on gender differences in STEM. We believe, however, that a fairer representation of the literature is warranted, especially considering the contentious nature of the topic.

\section{CONFOUNDERS AND STATISTICAL REANALYSIS}

In addition to selectively citing the literature, Strumia (2021) fails to consider the potential impact of a broad range of confounding factors on gender differences in citations and the publication process. To help highlight the problems this introduces, one of the authors $(\mathrm{EH})$ reanalyzed a subset of Strumia's data.

The reanalyzed data contain 5,599 solo-authored articles-5,386 authored by a man and 213 authored by a woman-published in five high-profile physics journals from 2010 to 2016 (inclusive). The selection criteria were designed to address the influence of key confounders in Strumia's data. First, we restricted the sample to solo-authored articles to account for the fact that male physicists are more likely to be senior authors on papers involving much larger research teams ${ }^{1}$. Second, restricting the data to articles published in a small set of well-known journals

\footnotetext{
1 In contrast, Strumia (2021), Hengel (2017), and Hengel and Moon (2020) assume that each coauthor on a paper contributes equally to it. This relationship, however, is unlikely to be linear when there are a very large number of coauthors, as there often are in fields such as high-energy experimental physics.
} 
made it easier to confirm the quality of gender assignment in Strumia's data ${ }^{2}$. By including journal-year fixed effects, we also better account for differing citation patterns between fields ${ }^{3}$. Third, younger articles have had less time to accrue citations and older articles are disproportionately by male authors. For that reason, we also restrict our analysis to newer articles (i.e., those published between 2010 and 2016) as well as controlling for journal-year fixed effects.

An additional difference between Strumia's study and our own is that we analyze data at the article level instead of aggregating citations over authors. This allows us to better address the following issues discussed in Hengel and Moon (2020):

1. Male authors disproportionately cluster at the very top and very bottom of the citation distribution, but raw citation counts are truncated from below at zero and unbounded from above. This generates a nonlinear mapping from quality onto citations that depends on the former's variance. When used as a proxy for quality, average citations for male-authored papers will, as a result, generally place too much weight on high-citation papers and not enough weight on low-citation papers compared to the average for female-authored papers. To deal with this issue, we transform raw citation counts with the inverse hyperbolic sine function (asinh). We stress, however, that our results do not meaningfully change if we use raw citation counts as the dependent variable instead.

2. Unobserved (or uncontrolled for) confounders (e.g., winning a prestigious award) boost citations conditional on quality and disproportionately correlate with articles and authors located in the distribution's right tail. A related concern is that the citations that a paper accumulates are not fixed in time. As a result, they could be influenced by the future success or failure of a paper's authors (i.e., even among nonsuperstar physicists, a stronger publishing record later on probably drives citations to earlier work, all else being equal). For evidence see, for example, Bjarnason and Sigfusdottir (2002). Both factors potentially correlate with gender: For example, women produce fewer papers than men and are proportionately less likely to win the Nobel Prize.

To deal with these issues, we control for the year in which an author was first published and her total number of lifetime publications ${ }^{4}$. Our results should therefore be interpreted as gender differences in citations between authors who began their careers around the same time and had accumulated similar lifetime "fame" at the time citations were collected.

Assuming citations are not perfectly explained by these variables ${ }^{5}$, gender differences present in Strumia's complete, unadjusted data should also be present in the conditioned, restricted

2 To verify gender, we manually searched (via Google) for all the previously identified women and the subset of men with no more than one citation. Gender was identified based on pronouns or inferred from photos. Authors classified as female about whom we found no information—or the information we did find was ambiguous about gender-are omitted from our analysis (13 observations). As per the main text, we note that we would rarely identify individuals as nonbinary from this gender analysis. From this analysis, we found that $21-26 \%$ of people classified as women are more reasonably classified as men. Moreover, their solo-authored articles tended to receive a disproportionately low number of citations.

3 Strumia (2021), in contrast, adjusts for field by normalizing a paper's citation count by the length of its own reference list, which roughly correlates with field (Strumia, 2021; Eq. 1). (We obtain similar results using his normalized indicator of citations.)

${ }^{4}$ To account for age, Strumia (2021) weights each author by one-half times the inverse of the proportion of authors of the same gender who first published in the same year he or she did (Eq. 2). For example, if 300 authors - two of whom were female-first published in 1995, then each female author would be weighted by 75 , whereas each male author would be weighted by roughly 0.5 .

${ }^{5}$ Effectively, the distribution of citations cannot collapse to a degenerate distribution after conditioning on these variables. As evidence against this possibility, it does not appear that citations are homogenous, conditional on, for example, journal. 

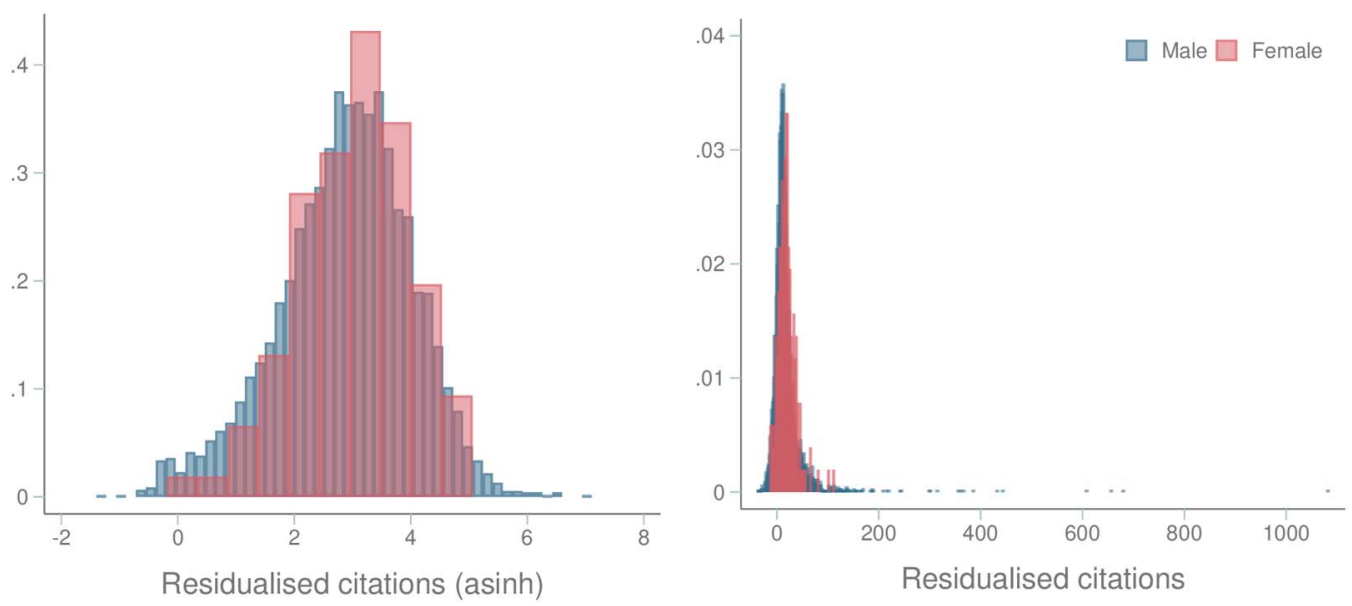

Figure 1. Distribution of citations for solo-authored papers. Note. Graphs display histograms of asinh transformed (left) and raw (right) citations for solo-authored papers by men (blue) and women (pink) published between 2010 and 2016 (inclusive) in Physical Review D, Astrophysical Journal, Journal of High Energy Physics, Physical Review Letters, and Physics Letters B. Citations have been residualized with respect to year-journal fixed effects, fixed effects for each author's year of first publication, and total lifetime number of publications. Data from Strumia (2021).

data if male physicists are, in fact, biologically more productive or produce higher quality work than women ${ }^{6}$. In other words, if male talent is more variable, then male physicists' work will be (on average) higher "quality" (as defined by citation count), all else being equal. As a result, a paper by a famous female physicist published in 2010 in Physical Review D should be (on average) cited less than a paper by a similarly famous male physicist that was also published in Physical Review D in 2010.

This is not what we observe. Our evidence suggests that female-authored papers receive about $12 \log$ points more citations than male-authored papers, conditional on covariates. This figure is weakly statistically significant.

Figure 1 shows the distribution of citations among male vs. female-authored papers in this sample for both transformed and nontransformed citations. Note that the distribution of citations to male-authored papers closely overlaps with the distribution of citations to female-authored papers across the entire range of the distribution.

Estimated gender differences in citations at the mean for each of the five journals are shown in Figure 2 . They consistently suggest either no statistically significant gender gap in citations or a citation gap that favors women.

We are aware that automatic and binary classification here can be problematic (Keyes, 2018), especially for nonbinary and transgender physicists. We have followed Strumia (2021)'s use of a binary classification scheme, as we wish to highlight issues with the original analysis and we do not have access to demographic information provided by the authors within the present work. But we recognize the shortcomings of a reductive analysis to an apparent gender binary in such bibliometric analyses and discussions (Strauss, Borges et al., 2020; Rasmussen, Maier et al.,

\footnotetext{
${ }^{6}$ Assuming male and female talent is normally distributed with identical means, then gender differences in variability are equivalent to gender differences in (conditional) averages. Presumably, all physicists are drawn from the top half of each distribution; thus, greater variability in men implies that average male talent is higher than average female talent, conditional on being a physicist.
} 


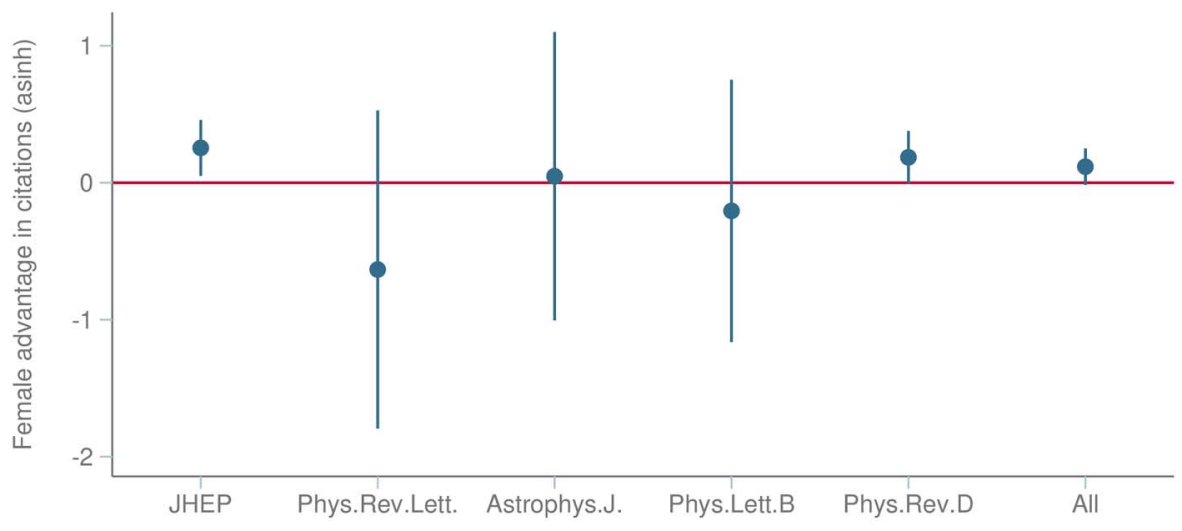

Figure 2. Gender differences in citations across journals. Note. The first five figures display conditional mean gender differences in citations (asinh); a positive value indicates female-authored papers are cited more than male-authored papers, conditional on included controls. They are estimated by regressing citations (asinh) on a dummy variable equal to 1 if the author was female in the subsamples of solo-authored articles published between 2010 and 2016 (inclusive) in Journal of High Energy Physics (JHEP), Physical Review Letters (Phys.Rev.Lett), Astrophysical Journal (Astrophys. J.) and Physical Review D (Phys.Rev.D). The final figure is the estimated gender difference in the pooled data. Lines represent $95 \%$ confidence intervals from standard errors clustered on an author's year of first publication. All estimates control for fixed effects for year or year-journal interactions, year of first publication, and total lifetime number of publications. Data from Strumia (2021).

2019) and urge journals to collect demographic data regarding authorship more routinely and to promote the use of self-identity.

\section{THE HIGHER MALE VARIABILITY HYPOTHESIS}

Despite the admission in Strumia (2021) that the simple interpretation laid out therein "can easily be incomplete," the data are nonetheless explained in the context of the highly contentious higher male variability (HMV) hypothesis and a biological basis of difference, together with gendered differences in interests. Once again, there is a lack of appropriate representation and citation of the relevant extensive literature base.

The HMV hypothesis is widely contested and debated; both Gray, Lyth et al. (2019) and Stevens and Haidt (2017) provide systematic and even-handed discussions. Note, in particular, the geographical variation highlighted by Gray et al. (2019) in relation to the HMV hypothesis, which counters the claims that any observed gender differences are biological in origin:

... we find that there is significant heterogeneity between countries, and that much of this can be quantified using variables applicable across these assessments (such as test, year, malefemale effect size, mean country size, and Global Gender Gap Indicators).

Geographical and temporal heterogeneity are consistently observed in a variety of measures of gender disparity in STEM (see, for example, Breda, Jouini, \& Napp, 2018; Kane \& Mertz, 2012; Nollenberger, Rodríguez-Planas, \& Sevilla, 2016). Counterintuitively, however, the so-called "gender equality paradox" put forward by Stoet and Geary (2018), and cited on a number of occasions in Strumia (2021), is the claim that countries with a higher level of gender equality tend to have less gender balance in STEM fields. We note that Stoet and Geary's arguments have been undermined significantly by the many deficiencies in their data analysis highlighted by Richardson, Reiches et al. (2020) (including those that have necessitated the publication of a corrected version of Stoet and Geary [2018]). 
Strumia's undue and unwarranted confidence in the HMV interpretation-given his admission that the data are influenced by "questionable systematic issues" - is such that the abstract closes with the claim that the "quantitative shape" of the data can be "fitted by higher male variability." As highlighted by Hyde (2014),

... even if there is slightly greater male variability for some cognitive measures, this finding is simply a description of the phenomenon. It does not address the causes of greater male variability, which could be due to biological factors, sociocultural factors, or both.

The sociocultural factors to which Hyde refers are exceptionally difficult to model (and correct for) yet may play an integral role, as discussed by Kalender, Marshman et al. (2019) in their study of gendered patterns in the construction of physics identity.

Strumia (2021) also does not provide any direct evidence for the causal link he suggests between the HMV hypothesis, biological determinism, and citation rates. Instead, there is nothing more than an inference based on drawing a comparison with what are termed "psychometric observations." This is an entirely unjustified conflation of correlation and causation and has no place in a rigorous interpretation of the data. Only properly controlled studies allow for a robust distinction between correlation and causation-a fundamental tenet of all statistical analysis. Strumia (2021) admits that those controlled studies are simply not possible.

Similarly, the homogeneity of the people/things dimension (Spelke, 2005; Thelwall, Bailey et al., 2019) is very much overstated in Strumia (2021) in support of the argument that a lack of interest underpins the level of participation by women in HEP. Thelwall et al. (2019), whose work is cited by Strumia in the context of the people/things metric, devote a great deal of time in the conclusion of their paper highlighting the deficiencies in their approach:

Thus, the people/things dimensions can only provide a partial explanation for gender differences in topic choices across the full spectrum of academia because there are many important exceptions ... Given that the current research has not attempted to assess any cause and effect relationships, deviations from the people/thing dimensions could also be due to other factors within academia that deflect people from pursuing their interests, such as editorial, departmental or funding policies.

None of this important yet difficult-to-quantify nuance is captured by the discussion in Strumia (2021).

Another confounding factor is vocal criticism of women within academia by individuals such as Strumia, who may well be contributing to the problem of women feeling unwelcome in physics. As Halpern, Benbow et al. (2007), whose paper is cited in Strumia (2021), put it,

We conclude that early experience, biological factors, educational policy, and cultural context affect the number of women and men who pursue advanced study in science and math and that these effects add and interact in complex ways. There are no single or simple answers to the complex questions about sex differences in science and mathematics.

The issues outlined above demonstrate that extreme care must be taken when arguing for causal relationships among variables in a system where confounders are exceptionally difficult to deal with (or, indeed, to identify in the first place). A full, appropriately controlled statistical analysis of the cumulative influence of bias-both explicit and implicit, including differing levels of scrutiny in the publication process (Hengel, 2017), discrimination, harassment, bullying, parental and 
domestic responsibilities, access to research funding, and variations in teaching, committee, and pastoral care workloads on the work of women in HEP (and hence on their outputs and citation rates)—would be needed to interpret Strumia's observations in a scientifically robust and objective manner.

\section{BIBLIOMETRICS AS A PROXY FOR SCIENTIFIC QUALITY}

There is yet a broader issue surrounding Strumia's methodology: The analysis implicitly assumes not only a direct link between citation rates (normalized as per Eq. 1 of Strumia [2021]) and scientific quality, but, as noted above, it infers - with no direct quantitative evidence- $a$ further link to the HMV hypothesis. Moreover, it is assumed that all authors contribute equally to a paper. Strumia recognizes, to some extent, that this assumption of equal contributions is problematic- “... there is no warranty that each author contributed to each paper" — but his justification is very far from compelling:

Despite this, the data show that the total fractionally counted bibliometric output of collaborations scales, on average, as their number of authors [Rossi, Strumia, \& Torre (2019)], suggesting that large collaborations form when scientifically needed and that gift authorship does not play a large role.

A lack of gift authorship (i.e., the inclusion of an author who contributed little or nothing at all) is a far cry from the extreme assumption of equal contributions across the entire authorship of a paper. (This is another justification for the restriction of our analysis in the previous section to solo-authored publications.)

In dismissing the role of sociological factors, Strumia (2021) also subjectively (and rather inconsistently) appeals to the reader's perception of the prestige of top authors: "A physicist might read their names and conclude that no sociological confounder can wash away most of them." MacRoberts and MacRoberts (2010) describe the process of biased citing, which involves a number of considerations including the "halo effect" (exemplified by the preceding quote from Strumia [2021]), in-house citations, obliteration, and the Matthew effect. Each of these effects, and others (Cowley, 2015) can lead to disproportionate citations of the primary literature. There is also very good evidence that seminal papers can often be cited but not actually read (Ball, 2002; Simkin \& Roychowdhury, 2003).

More broadly, the use of citations as a measure of scientific quality is highly questionable and has been the subject of significant debate for decades (see Leydesdorff, Bornmann et al. (2016) for a review). We note that Strumia highlights that "traditional metrics (such as citation counts, $h$-index, paper counts) now fail to provide reasonable proxies for scientific merit in fundamental physics." His solution-the introduction of what he terms an "individual citation" metric, Eq. 1 of Strumia (2021)—is not a compelling strategy to isolate scientific quality from citation numbers. As Aksnes, Langfeldt, and Wouters (2019) highlight,

Research quality is a multidimensional concept, where plausibility/soundness, originality, scientific value, and societal value commonly are perceived as key characteristics ... citations reflect aspects related to scientific impact and relevance, although with important limitations ... there is no evidence that citations reflect other key dimensions of research quality

Strumia's reasoning regarding the efficacy of citation metrics as a measure of scientific quality is also notably circular: "... bibliometric indicators, that can be used as reliable proxies for scientific merit, being significantly correlated to human evaluations such as scientific prizes." The 
attempt to draw a distinction here between bibliometric indicators and "human evaluations such as scientific prizes" is telling. Bibliometrics are not a wholly objective quantitative proxy for scientific merit-they are just as much a human evaluation as is a scientific prize. The award of scientific prizes very often involves detailed consideration of citation rates, and the prestige that stems from a scientific prize will in turn drive more citations for a particular scientist. It is naïve to imagine that bibliometrics are an independent measure of scientific merit whose usage is somehow justified by their correlation with the award of "human evaluations such as scientific prizes."

\section{CONCLUSIONS}

The analysis presented in Strumia (2021) suffers from a number of deficiencies that severely undermine the inferences drawn and the conclusions reached therein. In particular, the contributions of a wide variety of potential confounding factors are not only unaddressed but are dismissed with no justification. This unwarranted dismissal, coupled with a fundamental confusion of correlation and causation when drawing inferences, is especially problematic in a study that claims to provide an unbiased assessment of the role of gender in citation patterns. Instead, the analysis throughout is very far from neutral or disinterested; the data are interpreted within an ideologically motivated context-as is clear from both the paper itself and previous work by the same author, namely Strumia (2018) and Strumia (2019).

In that broader context, Strumia's analysis is substantively problematic. The views he espouses have had an impact not only across the physics and STEM communities but have been widely reported across the international media (including Conradi, 2019; Giuffrida and Busby, 2018; Nicholson, 2018; Young, 2019). Physics as a discipline is broadly acknowledged to struggle with both the recruitment and retention of women (Eddy \& Brownell, 2016; Kalender et al., 2019; Porter \& Ivie, 2019). The widespread dissemination of derogatory and unsubstantiated views about women's ability in this sphere is not going to overcome this problem. It will be off-putting to talented women who might be considering a career in the sciences and contributes to the hostile environment endured by many women (and other minorities) currently working in physics. There is an increasing body of research that suggests that an individual's performance in an academic context can be harmed by an awareness that others' perception of their work might be distorted by stereotypes (see, for example, Casad, Hale, \& Wachs, 2017; Kalender et al., 2019; Shapiro \& Williams, 2012). The associated cultural implications can result in minoritized individuals not contributing or disengaging from their academic communities.

Ultimately, the work presented in Strumia (2021) is not merely a flawed, biased, and ideologically motivated analysis. It is also likely to be actively harmful to the progress of women in physics, to the detriment not only of many individuals but of our entire community.

\section{ACKNOWLEDGMENTS}

We thank Beck Strauss for helpful discussions regarding issues associated with misclassification of gender.

\section{FUNDING INFORMATION}

TBB acknowledges funding of his Research Fellowship from the Royal Academy of Engineering. PM similarly acknowledges the Engineering and Physical Sciences Research Council (EPSRC) for the award of an established career fellowship (EP/T033568/1.). 


\section{DATA AVAILABILITY}

Data and replication files for all analyses presented in this paper are available on GitHub (https:// github.com/erinhengel/strumia-qss).

\section{REFERENCES}

Aksnes, D. W., Langfeldt, L., \& Wouters, P. (2019). Citations, citation indicators, and research quality: An overview of basic concepts and theories. SAGE Open, 9(1), 1-17. DOI: https://doi.org /10.1177/2158244019829575

Ball, P. (2002). Paper trail reveals references go unread by citing authors. Nature, 420(6916), 594. DOI: https://doi.org/10.1038 /420594a, PMID: 12478249

Bjarnason, T., \& Sigfusdottir, I. D. (2002). Nordic impact: Article productivity and citation patterns in sixteen Nordic sociology departments. Acta Sociologica, 45(4), 253-267. DOI: https:// doi.org/10.1177/000169930204500401

Breda, T., Jouini, E. \& Napp, C. (2018). Societal inequalities amplify gender gaps in math. Science, 359(6381), 1219-1220. DOI: https://doi.org/10.1126/science.aar2307, PMID: 29590066

Burns, K. E. A., Straus, S. E., Liu, K., Rizvi, L., \& Guyatt, G. (2019). Gender differences in grant and personnel award funding rates at the Canadian Institutes of Health Research based on research content area: A retrospective analysis. PLOS Medicine, 16(10), e1002935. DOI: https://doi.org/10.1371/journal.pmed.1002935, PMID: 31613898, PMCID: PMC6793847

Caplar, N., Tacchella, S., \& Birrer, S. (2017). Quantitative evaluation of gender bias in astronomical publications from citation counts. Nature Astronomy, 1, 0141. DOI: https://doi.org/10.1038 /s41550-017-0141

Card, D., DellaVigna, S., Funk, P., \& Iriberri, N. (2020). Are referees and editors in economics gender neutral? Quarterly Journal of Economics, 135(1), 269-327. DOI: https://doi.org/10.1093/qje /qjz035

Casad, B. J., Hale, P., \& Wachs, F. L. (2017). Stereotype threat among girls: Differences by gender identity and math education context. Psychology of Women Quarterly, 41(4), 513-529. DOI: https://doi.org/10.1177/0361684317711412

Conradi, P. (2019). Alessandro Strumia: The data doesn't liewomen don't like physics. The Times, March 24. https://www .thetimes.co.uk/article/alessandro-strumia-the-data-doesnt-lie -women-dont-like-physics-jlobpfd9t (accessed March 18, 2020).

Cowley, S. J. (2015). How peer-review constrains cognition: On the frontline in the knowledge sector. Frontiers in Psychology, 6, 1706. DOI: https://doi.org/10.3389/fpsyg.2015.01706, PMID: 26579064, PMCID: PMC4630500

Del Giudice, M., Puts, D. A., Geary, D. C., \& Schmitt, D. P. (2019). Sex differences in brain and behavior: Eight counterpoints. Psychology Today, April 8. https://www.psychologytoday.com /intl/blog/sexual-personalities/201904/sex-differences-in-brain -and-behavior-eight-counterpoints?amp (accessed March 18, 2020).

Dworkin, J. D., Linn, K. A., Teich, E. G., Zurn, P., Shinohara, R. T., \& Bassett, D. S. (2020). The extent and drivers of gender imbalance in neuroscience reference lists. Nature Neuroscience, 23, 918-926. DOI: https://doi.org/10.1038/s41593-020-0658-y, PMID: 32561883

Eddy, S. L., \& Brownell, S. E. (2016). Beneath the numbers: A review of gender disparities in undergraduate education across science, technology, engineering, and math disciplines. Physical Review Physics Education Research, 12(2), 020106. DOI: https://doi.org/10.1103/PhysRevPhysEducRes.12.020106
Fox, C. W., \& Paine, C. E. T. (2019). Gender differences in peer review outcomes and manuscript impact at six journals of ecology and evolution. Ecology and Evolution, 9(6), 3599-3619. DOI: https://doi.org/10.1002/ece3.4993, PMID: 30962913, PMCID: PMC6434606

Giuffrida, A. \& Busby, M. (2018). 'Physics was built by men': Cern suspends scientist over remarks. The Guardian, October 1. http:// www.theguardian.com/science/2018/oct/01/physics-was-built -by-men-cern-scientist-alessandro-strumia-remark-sparks-fury (accessed March 18, 2020).

Gray, H., Lyth, A., McKenna, C., Stothard, S., Tymms, P., \& Copping, L. (2019). Sex differences in variability across nations in reading, mathematics and science: A meta-analytic extension of Baye and Monseur (2016). Large-Scale Assessments in Education, 7(1), 2. DOI: https://doi.org/10.1186/s40536-019-0070-9

Halpern, D. F., Benbow, C. P., Geary, D. C., Gur, R. C., Hyde, J. S., \& Gernsbacher, M. A. (2007). The science of sex differences in science and mathematics. Psychological Science in the Public Interest, 8(1), 1-51. DOI: https://doi.org/10.1111/j.1529 -1006.2007.00032.x, PMID: 25530726, PMCID: PMC4270278

Helmer, M., Schottdorf, M., Neef, A., Battaglia, D. (2017). Gender bias in scholarly peer review. eLife, 6, e21718. DOI: https://doi org/10.7554/eLife.21718, PMID: 28322725 , PMCID: PMC5360442

Hengel, E. (2017). Publishing while female. Are women held to higher standards? Evidence from peer review. Cambridge Working Papers in Economics 1753. Faculty of Economics, University of Cambridge. http://www.erinhengel.com/research /publishing female.pdf.

Hengel, E., \& Moon, E. (2020). Gender and quality at top economics journals. Working Paper in Economics 202001. University of Liverpool Management School. https://erinhengel.github.io /Gender-Quality/quality.pdf.

Hyde, J. S. (2014). Gender similarities and differences. Annual Review of Psychology, 65, 373-398. DOI: https://doi.org/10 .1146/annurev-psych-010213-115057, PMID: 23808917

Kalender, Z. Y., Marshman, E., Schunn, C. D., Nokes-Malach, T. J., \& Singh, C. (2019). Gendered patterns in the construction of physics identity from motivational factors. Physical Review Physics Education Research, 15(2), 020119. DOI: https://doi.org/10 .1103/PhysRevPhysEducRes.15.020119

Kane, J. M., \& Mertz, J. E. (2012). Debunking myths about gender and mathematics performance. Notices of the AMS, 59(1), 10-21. DOI: https://doi.org/10.1090/noti790

Keyes, O. (2018). The misgendering machines: Trans/HCl implications of automatic gender recognition. Proceedings of the ACM on Human-Computer Interaction, Vol. 2, No. CSCW, Article 88. DOI: https://doi.org/10.1145/3274357

Leydesdorff, L., Bornmann, L., Comins, J. A., \& Milojević, S. (2016). Citations: Indicators of quality? The impact fallacy. Frontiers in Research Metrics and Analytics, 1, 787. DOI: https://doi.org/10 .3389/frma.2016.00001

MacRoberts, M. H. \& MacRoberts, B. R. (2010). Problems of citation analysis: A study of uncited and seldom-cited influences. Journal of the American Society for Information Science and Technology, 61(1), 1-12. DOI: https://doi.org/10.1002/asi.21228 
Nicholson, L. (2018). Physics does have a women problem-and it's not rocket science to work out why. Daily Telegraph, October 1. https://www.telegraph.co.uk/women/work/physics -does-have-women-problem-not-rocket-science-work/ (accessed March 18, 2020).

Nollenberger, N., Rodríguez-Planas, N., \& Sevilla, A. (2016) The math gender gap: The role of culture. American Economic Review, 106(5), 257-261. DOI: https://doi.org/10.1257/aer .p20161121

Porter, A. M., \& Ivie, R. (2019). Women in physics and astronomy, 2019. American Institute of Physics. https://www.aip.org/sites /default/files/statistics/women/Women\%20in\%20Physics\%20 and\%20Astronomy\%202019.1.pdf.

Rasmussen, K. C., Maier, E., Strauss, B. E., Durbin, M., Riesbeck, L., ... Erena, A. (2019). The nonbinary fraction: Looking towards the future of gender equity in astronomy. Bulletin of the AAS, 51(7).

Richardson, S. S., Reiches, M. W., Bruch, J., Boulicault, M., Noll, N. E., \& Shattuck-Heidorn, H. (2020). Is there a gender-equality paradox in science, technology, engineering, and math (STEM)? Commentary on the study by Stoet and Geary (2018). Psychological Science, 31(3), 338-341. DOI: https://doi.org/10 .1177/0956797619872762, PMID: 32043923

Royal Society of Chemistry. (2019). Is publishing in the chemical sciences gender biased? Royal Society of Chemistry. https://www .rsc.org/globalassets/04-campaigning-outreach/campaigning /gender-bias/gender-bias-report-final.pdf.

Shapiro, J. R., \& Williams, A. M. (2012). The role of stereotype threats in undermining girls' and women's performance and interest in STEM fields. Sex Roles, 66(3-4), 175-183. DOI: https://doi.org /10.1007/s11199-011-0051-0

Simkin, M. V., \& Roychowdhury, V. P. (2003). Read before you cite! Complex Systems, 14(3), 269-274.

Spelke, E. S. (2005). Sex differences in intrinsic aptitude for mathematics and science? A critical review. American Psychologist, 60(9), 950-958. DOI: https://doi.org/10.1037/0003-066X 60.9.950, PMID: 16366817

Steinberg, J. J., Skae, C., \& Sampson, B. (2018). Gender gap, disparity, and inequality in peer review. The Lancet, 391(10140),
2602-2603. DOI: https://doi.org/10.1016/S0140-6736(18) 31141-3

Stevens, S. \& Haidt, J. (2017). The Google memo: What does the research say about gender differences? Heterodox: The Blog, August 10. https://heterodoxacademy.org/the-google-memo -what-does-the-research-say-about-gender-differences/ (accessed March 18, 2020).

Stoet, G., \& Geary, D. C. (2018). The gender-equality paradox in science, technology, engineering, and mathematics education. Psychological Science, 29(4), 581-593. DOI: https://doi.org /10.1177/0956797617741719, PMID: 29442575

Strauss, B. E., Borges, S. R., Faridani, T., Grier, J. A., Kiihne, A., ... Zamloot, V. (2020). Nonbinary systems: Looking towards the future of gender equity in planetary science. arXiv, arXiv:2009 .08247 .

Strumia, A. (2018). Experimental test of a new global discrete symmetry. https://alessandrostrumiahome.files.wordpress.com/2019 /03/strumiagenderslidescern.pdf.

Strumia, A. (2019). Is there a gender bias in physics? https:// alessandrostrumiahome.files.wordpress.com/2019/10 /genderslidesleiden19.pdf.

Strumia, A. (2021) Gender issues in fundamental physics: A bibliometric analysis. Quantitative Science Studies (this issue).

Thelwall, M., Bailey, C., Tobin, C., \& Bradshaw, N.-A. (2019). Gender differences in research areas, methods and topics: Can people and thing orientations explain the results? Journal of Informetrics, 13(1), 149-169. DOI: https://doi.org/10.1016/j.joi .2018 .12 .002

Witteman, H. O., Hendricks, M., Straus, S., \& Tannenbaum, C. (2019). Are gender gaps due to evaluations of the applicant or the science? A natural experiment at a national funding agency. The Lancet, 393(10171), 531-540. DOI: https://doi.org/10.1016 /S0140-6736(18)32611-4

Young, C. (2019). Alessandro Strumia: Another politically-correct witch-hunt, or a more complicated story? Quillette, April 22. https://quillette.com/2019/04/22/alessandro-strumia-another -politically-correct-witch-hunt-or-a-more-complicated-story/ (accessed March 18, 2020). 